\title{
What Shall We Do Now? Report of Unexpected CT Finding and Its Management
}

\author{
Capolupo GT, Mascianà $G^{*}$ and Caricato M \\ Institute of Geriatric Surgery, Università Campus Bio-Medico, Italy
}

\begin{abstract}
A 41-years-old patient underwent total colectomy and ileal pouch-anal anastomosis with derivative ileostomy for a familiar adenomatous polyposis coli in June 2008. Pathologic examination of the specimen showed a pT1 N1 adenocarcinoma of the rectum. The patient underwent chemo-radiation and subsequent adjuvant chemotherapy. A stenosis of the pouch-anal anastomosis developed. The patient underwent several endoscopic dilations. The ileostomy was taken down in March 2016. A recurrence of the stenosis and a peri-anastomotic collection were observed. An endoscopic drainage of the collection was performed on October $3^{\text {rd }}, 2016$. The endoluminal drainage was removed and the fistula closed with an OVESCO ${ }^{\circledast}$ clip on October $10^{\text {th }}$. The patient was re-admitted on October $19^{\text {th }}$ with serotine fever $\left(37.8^{\circ} \mathrm{C}\right)$ without leucocytosis. An endoscopic examination of the pouch showed absence of purulent discharge but many fistulous orifices around the pouch-anal anastomosis were detected. A CT scan showed little peri-anastomotic collections (Figure 1) with air-fluid levels and massive hepatic portal venous gas (Figure 2). The patient was in good clinical conditions, apiretic, blood tests showed: WBC: $4290 / \mu \mathrm{L}, \mathrm{PCT}: 0.10 \mathrm{ng} / \mathrm{ml}$.
\end{abstract}

\section{Keywords}

Portal system, Endoscopy/gastrointestinal, Intestinal fistula

\section{What is the Cause of the Portal Pneumatosis?}

Finding of a relevant amount of gas in portal venous system has traditionally been associated with serious clinical conditions with poor outcome, as it happens in abdominal abscess or intestinal infarction [1]. In some cases, venous hepatic gas is an incidental finding with scarce clinical relevance: It has been described as a consequence of diagnostic or therapeutic invasive procedures [2] such as surgery, hepatic artery embolization, operative endoscopic procedures [3]. In our case, an abdominal abscess was present but clinical serious signs of sepsis were absent. Based on clinical findings, antibiotic treatment with meropenem (500 mg tid i.v.) was administered without further diagnostic or therapeutic interventions, assuming that the portal pneumatosis was due to the endoscopic examination. One day later a new CT scan detected a marked reduction of hepatic portal venous gas (Figure 3); the patient resumed oral feeding and three days later was discharged. Our experience confirm that hepatic portal venous gas can be related to endoscopic procedure; thus, it can be managed on the basis of patient's general clinical conditions, and in selected cases it will disappear without therapeutic interventions with a good outcome.

\section{Acknowledgements}

Dr. G. Capolupo and Dr. G. Masciana Patient care and management; image contribution Prof. M. Caricato: Revision

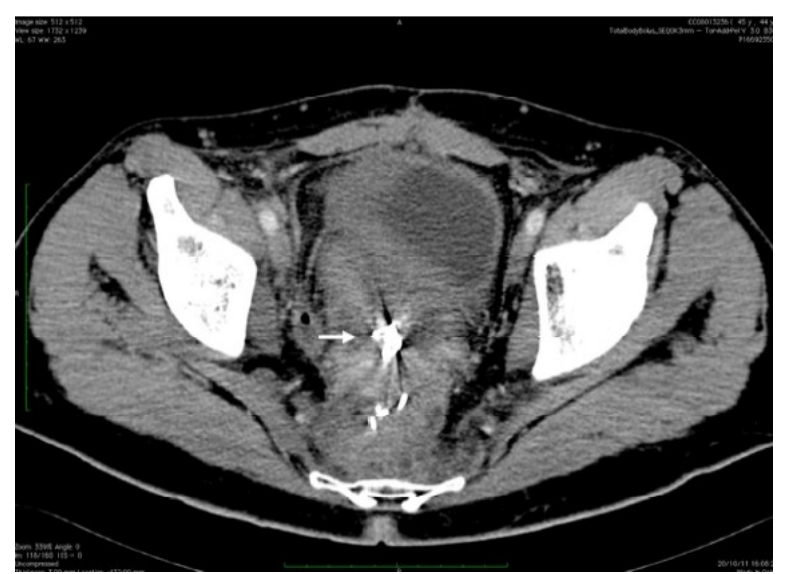

Figure 1: A CT scan showed little peri-anastomotic collections with air-fluid levels.

*Corresponding author: Mascianà Gianluca, Università Campus Bio-Medico, Via Alvaro del Portillo 21 - 00128 Rome, Italy, Tel: (+39)-06-22541-1428, Fax: (+39)-06-22541-1908

Accepted: June 22, 2019

Published online: June 24, 2019

Citation: Capolupo GT, Mascianà G, Caricato M (2019) What Shall We Do Now? Report of Unexpected CT Finding and Its Management. J Gastrointest Hepat Surg 2(1):16-17 


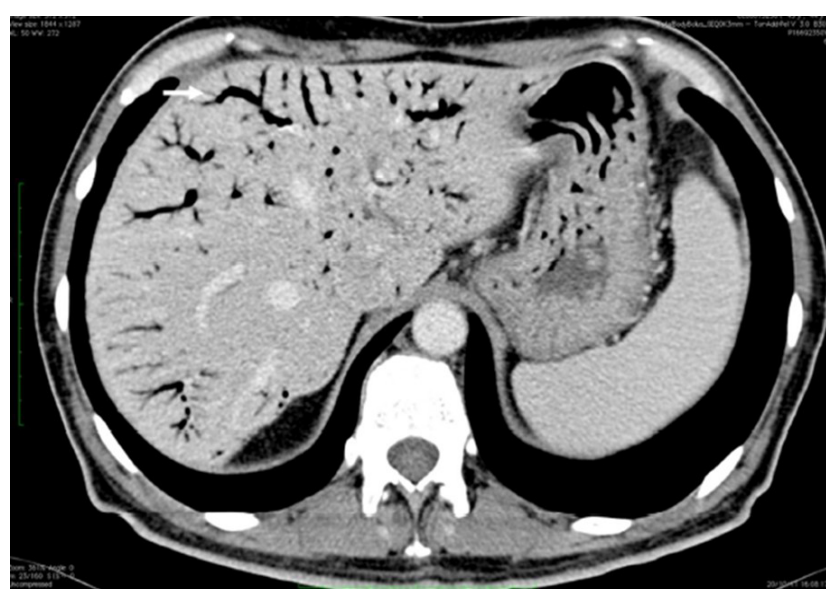

Figure 2: Unexpected CT finding: relevant amount of air in medium and small branches of portal vein (arrow) $254 \times 177 \mathrm{~mm}$ $(96 \times 96 \mathrm{DPI})$.

and final approval of the manuscript. This work received no specific funding.

\section{References}

1. Liebman PR, Patten MT, Manny J, et al. (1978) Hepatic-portal venous gas in adults: etiology, pathophysiology and clinical significance. Ann Surg 187: 281-287.

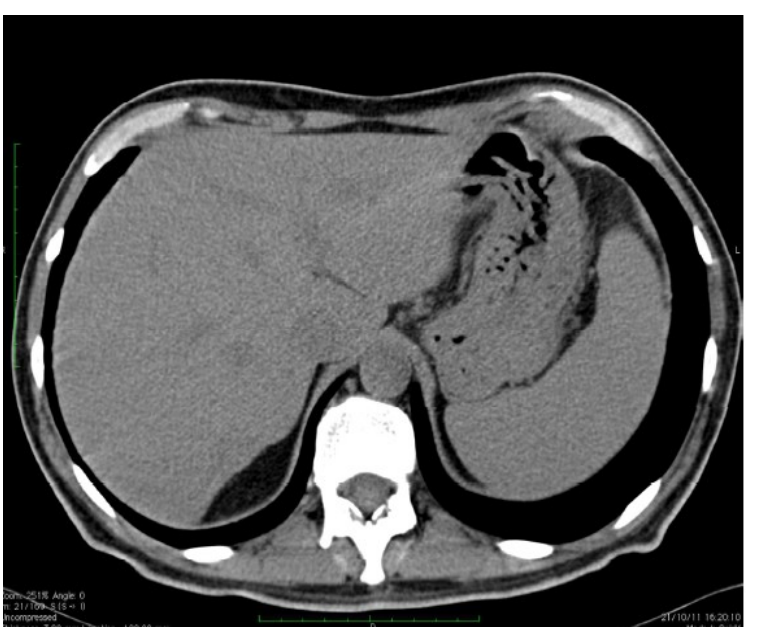

Figure 3: A CT scan detected a marked reduction of hepatic portal venous gas.

2. Shah PA, Cunningham SC, Morgan TA, et al. (2011) Hepatic Gas: Widening Spectrum of Causes Detected at CT and US in the Interventional era. Radiographics 31: 1403-1413.

3. Huycke A, Moeller DD (1985) Hepatic portal venous gas after colonoscopy in granulomatous colitis. Am J Gastroenterol 80: 637-638.

DOI: $10.36959 / 879 / 375$ 